\title{
The Association of Clinical Pathologists: 89th general meeting
}

The 89th general meeting of the Association of Clinical Pathologists was held at Imperial College, London, on 28 and 29 September 1972 under the Presidency of Sir James Howie, whose Presidential Address is printed on page 921. On this occasion there were five symposia: 'The lymphoreticular system', 'Decompression sickness', 'Chromosomes and disease', 'Encephalitis and meningitis', and 'Equipment for haematology'. Abstracts of papers read at these meetings and at the other sessions follow.

\section{Scientific communications I}

\section{A Histological Analysis of Granulomatous Hepatitis} M. HUGHeS AND H. FOX (Department of Pathology, University of Manchester) A study has been made of 33 cases of granulomatous hepatitis. Of these, 12 were eventually established as cases of sarcoidosis, nine as cases of tuberculosis, and four as examples of primary biliary cirrhosis. No clinical diagnosis was ever reached in six cases.

Analysis of the histological features of these cases shows that the presence of caseation or of tubercle bacilli will only allow for the diagnosis of approximately $50 \%$ of cases of tuberculosis. Histological features which are suggestive of tuberculosis are a paucity of granulomata, a scantiness of giant cells, a relatively mild surrounding chronic inflammatory cell infiltrate and an absence of reticulin. The granulomata are more likely to be due to sarcoidosis if they are numerous, contain many giant cells, are confined to the portal areas, and show a well marked surrounding non-specific chronic inflammatory cell infiltrate.

Histological features of cases in which no clinical diagnosis was reached suggest that they are more likely to be due to sarcoidosis than to tuberculosis.

\footnotetext{
A Case of Brucella Arthritis in the Wrist J. L. BRENNAN AND C. A. MORRIS (Robert Jones and Agnes Hunt Orthopaedic Hospital, Oswestry and Public Health Laboratory, Shrewsbury) A farmer aged 48 presented in August 1971 with a history of pain in the right wrist for six weeks. There was swelling of the dorsum and radial side of the wrist. A provisional diagnosis of tenosynovitis was made and the wrist was immobilized in plaster for six weeks. In October the patient was discharged as he was by then free of pain and swelling, but he returned in February 1972 with a recurrence.
}

Radiographs of the wrist, which previously had shown no obvious bony abnormality, now revealed a destructive lesion of the hamate bone. Tuberculosis, rheumatoid arthritis, and brucellosis were considered in the differential diagnosis. The Brucella abortus agglutination test was positive in phenol saline and in mercaptoethanol at $1 / 400$ and the Brucella CFT positive at $1 / 320$.

The wrist was explored surgically. A synovial biopsy showed subacute inflammation with appearances intermediate between an acute pyogenie infection and classical tuberculosis. Typical epf thelioid systems and caseation were absent, but is defined epithelioid cells and one Langhans type giant cell were seen. The bone showed similar tissue in the marrow spaces with osteoclastic activity. Brucella abortus biotype 1 was cultured from the synovium. A diagnosis of brucella osteomyelitis and synovitis was made. The patient gave a history of brucellosis among his cattle.

Helminthic Pseudotumours of the Bowel P. P. ANTHONY (Middlesex Hospital Medical School, London) Human infestation with nematode worms of the superfamily Strongyloidea may give rise to serious surgical complications. In this communication 34 cases are presented from Uganda where the condition appears to be common but it is also known to occur in other parts of Africa, the Far East, and the Americas. Worms of the genus Oesophagostomum are most frequently responsible. These are common parasites of ruminants, apes, and monkeys in which their histotropic phase is confined to the $\tilde{O}$ bowel wall. Development in man is abnormal and N results in persistence and further migration of the 0 parasite outside the bowel wall. Large tumour-like masses and abscesses form, most commonly in the $\stackrel{\overparen{D}}{\overparen{D}}$ ileocoecal angle, where they are usually misdiagnosed $\stackrel{\mathcal{Q}}{+}$ as carcinoma, tuberculosis, or appendicular abscess, 7 particularly in expatriate Europeans. All ages and 
both sexes are equally affected. Symptoms are malaise, low fever, and abdominal pain. A mass is usually felt. Twenty-two of these 34 patients underwent major bowel resection because of uncertain or mistaken clinical diagnosis, including three of six Europeans who were thought to havecarcinoma of the caecum. Three characteristic pathological presentations may be distinguished: (1) In the acute phase a worm track is found leading to an abscess in or near the bowel wall. The contents are thick, odourless, sterile pus, and, often, a live parasite. (2) After some weeks the worm dies, the cavity is walled off and the histological appearances come to resemble caseating tuberculosis quite closely. (3) Eventually a fibrous mass results in which eosinophils remain prominent. It is likely that with increasing numbers of people travelling to, and returning from, the tropics these helminthic pseudotumours (helminthomas) will have to be considered in the differential diagnosis of intraabdominal masses and inflammatory conditions.

The Measurement of Circulating Ferritin A. JACOBS, M. R. BEAMISH, AND M. ALLISON (University Hospital of Wales, Cardiff) Ferritin can be measured in serum by immunometric essay. The mean level in normal men is $69 \mathrm{ng} / \mathrm{ml}$ and in normal women $35 \mathrm{ng} / \mathrm{ml}$. Patients with iron-deficiency anaemia have concentrations below $10 \mathrm{ng} / \mathrm{ml}$ and these levels are always associated with iron deficient erythropoiesis.

The concentration of ferritin in serum gives a quantitive measure of storage iron both in normal subjects and those with iron overload. In patients with Hodgkin's disease the fall in the serum iron concentration is associated with a rise in the serum ferritin levels.

\section{Symposium I}

\section{The lymphoreticular system}

The Lymphoreticular System: Morphological Organization KRISTIN HENRY (Department of Pathology, Royal Postgraduate Medical School, London) At both a functional and anatomical level lymphoreticular tissue can be horizontally divided into central or primary lymphoid organs such as thymus and the avian bursa of Fabricius, and peripheral or secondary lymphoid tissue such as lymph nodes, spleen, and gut-associated lymphoid tissue.Peripheral lymphoid tissue functions in an executive capacity subserving both cell-mediated immune reactions as well as antibody production, and also for clearing the blood, lymph, and tissue of particulate matter. Thus, these tissues are specifically organized and equipped for antigen capture and processing, for antibody production, and for non-specific, ie, nonimmunological, phagocytosis. Structural differences between these peripheral lymphoid tissues exists according to location, but all are characterized by their lymphocytic nature, the presence of primary lymphoid follicles and highly complex germinal centres, an intricate vasculature, and a framework of supporting cells and reticulin fibres between which are found the executive cells. According to their light- and ultrastructural features, the component nucleated cells of peripheral lymphoid tissues have been allocated to one of the following categories: (1) the lymphocyte series (T- and B-cell lines); (2) the dendritic (reticular) cells; (3) cells of the mononuclear phagocyte system; (4) endothelial cells; and (5) the supporting 'reticular' cells. The thymus differs in many respects from peripheral lympnoid tissue, but on a purely morphological basis can be distinguished by its unique lympho-epithelial structure and by a virtual absence of any morphological expression of local immune responsiveness. In the adult, bone marrow is the source of the stem cells for both central and peripheral tissues, and not the reticulum cell as previously supposed.

Lymphoreticular System: Functional Organization I. ROITT (Immunology Department, Middlesex Hospital Medical School, London) We now recognize two major lymphocyte populations, one dependent on the presence of the thymus gland ( $T$ cells) and the other controlled by the bursa or its equivalent in mammals (B cells). B lymphocytes mature to form the antibody-secreting cells, of which the plasma cell represents an end stage, and are therefore responsible for humoral immunity. T lymphocytes, when appropriately sensitized, confer a state of cellmediated immunity on the host which affords defence against certain intracellular facultative organisms such as tubercle and leprosy bacilli and certain viruses including those of the pox group. Although not themselves capable of secreting immunoglobulins, $T$ cells do cooperate with $B$ cells in the antibody response enabling them to be triggered more readily by antigen. The other important functional characteristic of $T$ lymphocytes is their ability to recognize and be activated by the surface antigens of viable 'foreign' cells of the same species and thence to transform into cytotoxic lymphoblasts capable of killing the target foreign cells in culture; this provides one important mechanism by which the host can reject homografts and presumably tumour cells which would be regarded as homografts if their specific neo-antigens were recognized as 'non-self'. Antibodies with specificities for surface markers on different lymphoid cells are providing increasing 